UM-P-92/93

\title{
Electromagnetic Deflection of Spinning Particles
}

\author{
John P. Costella and Bruce H. J. McKellar \\ School of Physics, The University of Melbourne, Parkville, Victoria 3052, Australia
}

(September 1992)

\begin{abstract}
We show that it is possible to obtain self-consistent and physically acceptable relativistic classical equations of motion for a point-like spin-half particle possessing an electric charge and a magnetic dipole moment, directly from a manifestly covariant Lagrangian, if the classical degrees of freedom are appropriately chosen. It is shown that the equations obtained encompass the well-tested Lorentz force and Thomas-Bargmann-Michel-Telegdi spin equations, as well as providing a definite specification of the classical magnetic dipole force, whose exact form has been the subject of recent debate. Radiation reaction - the force and torque on an accelerated particle due to its self-interaction - is neglected at this stage.
\end{abstract}

\section{INTRODUCTION}

The "classical limit" of mechanics has always played an important role in practical physics. While it may be regarded as preferable to describe the behaviour of a physical system by solving the problem exactly within quantum mechanics, in practice one need not always do so. If one is only interested in expectation values of operators, then one can make good use of Ehrenfest's theorem - which holds good under quite general circumstances (see, e.g., [1], §31) —and instead solve the equations of classical Hamiltonian or Lagrangian 
dynamics. Thus, for example, the Lorentz force law for charged particles, and the ThomasBargmann-Michel-Telegdi spin precession equation [2, 3] for particles with spin, are used to advantage every day —implicitly or explicitly —in a wide variety of practical situations.

These two examples, however, owe their widespread acceptance largely to an important quality they possess: they can be derived simply from a knowledge of the external electromagnetic multipolar fields of their point-like sources, regardless of the detailed structure of these sources 四. On the other hand, the force on a point-like particle with spin is not uniquely defined by the external properties of its dipolar electromagnetic fields; this fact, well noted by Thomas in 1927 [2], and more recently by Hraskó [4], is not yet universally appreciated. To obtain the "correct" force on (and, hence, complete equations of motion for) a particle with a magnetic moment, one must therefore make some assumptions, explicit or otherwise, about the nature of the object creating the dipolar field; it is this freedom that has contributed to the ongoing controversy on the subject [5].

It would be impossible to review here all of the various assumptions underlying previous attempts at a complete classical description of particles with spin; instead, we shall simply

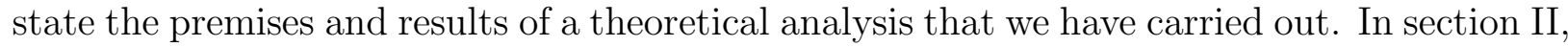
we outline our considerations in choosing a suitable relativistic classical Lagrangian for a particle with spin. We then, in section [II, outline our derivation of the equations of motion from this Lagrangian, and present the results in what we believe are the most transparent forms. As will be seen, the technique we shall use leads to the omission of all aspects

of radiation reaction - which is an omission that should be repaired - but nevertheless the results obtained do allow extensive contact with existing knowledge about the classical behaviour of particles with spin.

\section{AN APPROPRIATE LAGRANGIAN}

As already mentioned, some non-trivial assumptions must be made for one to obtain the force equation of motion for even a point-like particle endowed with a magnetic dipole 
moment. Our approach is to use a Lagrangian approach from the outset, and hence benefit from the abovementioned guarantee that Ehrenfest's theorem provides. This route is, however, still a potential minefield; as recently emphasised by Barut and Unal [10], the "spinning top" (or "current loop") phase space degrees of freedom are not appropriate to quantum spin-half particles. We shall return to this question shortly.

Our first consideration is to outline precisely what type of system we wish to address. In this specification we shall be exacting: we shall only be considering the intrinsic magnetic dipole moment of a spin-half, pointlike, structureless particle - together with any electric charge interaction it possesses, of course - in externally applied (but, in general, time- and space-varying) electromagnetic fields. It is only with such a tight restriction of our focus that we can proceed confidently in the classical realm at all, as will become apparent. For definiteness, we consider the electron a good approximation to the type of particle we are considering; however, we shall not claim any predictive control over contributions from the anomalous magnetic moment of the real electron, arising as it does from QED and other processes that are, in reality, additional degrees of freedom over those properly described by the single-particle Dirac equation. We shall not enter the debate as to whether a particle might "intrinsically" have a g-factor differing from 2 in the single-particle Dirac equation; if so, then such "anomalous" moments would be included in our considerations. In any case, since the pure Dirac moment of the electron dominates its anomalous moment numerically, our considerations will, at least, provide useful practical results for real electrons to leading order in the fine-structure constant.

Our main task in this section is to explain the particular Lagrangian that we have chosen to represent the magnetic interaction of such a particle. There are two distinct parts to this decision: firstly, the choice of the value that the Lagrangian should take; and, secondly, a determination of the appropriate classical degrees of freedom that are to be used in the Euler-Lagrange equations - and, hence, the functional form of the Lagrangian. The former choice is laid out for us: the Dirac equation gives us 


$$
L_{\mathrm{int}}=-\frac{g q \hbar}{8 m} \sigma^{\alpha \beta} F_{\alpha \beta},
$$

where $q$ is the charge of the particle ( $=-e$ for an electron) and $g$ is its gyromagnetic ratio (=2 for the simple minimally-coupled Dirac equation), and our units follow SI conventions with the exception that we set $\varepsilon_{0}=\mu_{0}=c=1$. However, to entertain the possibility of the single-particle Dirac equation fully describing a neutral particle with an anomalous moment, we shall replace the $g$ factor by the corresponding magnetic moment $\mu=g q \hbar / 4 m$, thereby separating the interaction of electric charge from that of magnetic moment, at least formally.

Our second task is infinitely more perilous: choosing an appropriate set of generalised coördinates to represent a spin-half particle. Firstly, we observe that the position of a particle is usually assumed to be an appropriate quantity in the classical limit; we shall also make this assumption. Thus, we take the (expectation value of the) four-position $z^{\alpha}(\tau)$ to be four of the independent degrees of freedom. (One can show that, in a general dynamical framework allowing particles with time-dependent masses, all four of these coördinates are indeed independent.) If we take the proper time for the particle, $\tau$, to be the generalised time of the classical Lagrangian framework, then the generalised velocities corresponding to the $z^{\alpha}$ are given by

$$
U^{\alpha} \equiv \frac{d z^{\alpha}}{d \tau} \equiv \dot{z}^{\alpha} .
$$

Having defined the four-velocity thus, we can then simply define the centre of energy frame for the particle as that instantaneous Lorentz frame in which $U^{\alpha}=(1,0,0,0)$.

We now turn to the magnetic moment of the particle. For a spin-half particle, the magnetic moment is parallel to the spin. In the centre of energy frame, we expect the particle's spin to be describable by a two-component spinor, of fixed magnitude $s=\frac{1}{2} \hbar$. The expectation value of this spinor is equivalent to a fixed-length three-vector, in this frame, whose direction describes simultaneously the expectation value of polarisation along any arbitrary axis, as well as the phase difference between the "up" and "down" components 
along such an axis, but which throws away the overall phase of the wavefunction (as does any reduction to the classical limit). It is this fixed-length three-vector that we shall assume to represent the magnetic moment of the particle.

Consider now the dynamical nature of spin angular momentum in the classical limit. It would seem, from the previous paragraph, that the spin should also be considered as a fixed-length three-vector in the centre of energy frame of the particle. However, this would preclude writing down the usual classical kinetic Lagrangian of rotational motion in terms of generalised velocities (i.e. the time derivatives of the Euler angles), namely, in the non-relativistic limit, [1]

$$
L=\frac{1}{2} \boldsymbol{\omega} \cdot \boldsymbol{I} \cdot \boldsymbol{\omega},
$$

and thereby preclude obtaining the torque on the particle by means of the Euler-Lagrange equations for the rotational degrees of freedom. Our approach, therefore, shall be the following: we shall consider the spin of the particle to be given, in the non-relativistic limit, by its usual form

$$
s=\boldsymbol{I} \cdot \boldsymbol{\omega},
$$

with the "kinetic" term ([3) retained in the Lagrangian in this same limit. This allows the magnitude of the spin, $|s|=s$ to be an a priori dynamical variable. (We know, of course, that $s$ should, ultimately, be a constant of the motion (i.e. $\left.\frac{1}{2} \hbar\right)$ if we are to accept the equations of motion as applicable to spin-half particles; we shall discuss this necessity in greater detail shortly.) We shall, on the other hand, still consider the magnetic moment of the particle to be formally a function of the generalised coördinates themselves (i.e. the Euler angles) and not the velocities. This distinction between the spin and magnetic moment of an electron is an unfamiliar concept within quantum mechanics; indeed, in that situation, no distinction need be made. However, this distinction is vital in the classical framework - at least, if one wants to derive the equations of motion from a Lagrangian - as the spin angular momentum and magnetic dipole moment of an arbitrary classical object are not, in general, related in 
any special way. Our ansatz that the magnetic moment is dependent on the Euler angles alone cannot be justified a priori; the only justification will be that it produces equations of motion that satisify the strict requirements of consistency for spin-half particles - that have not, to our knowledge, been satisfied by previous classical Lagrangian approaches. We further note, at this stage, that the ultimate parallelism of spin and magnetic moment does not bar us from considering them to have different functional dependencies: the fact that they are parallel may be introduced, in this classical context, as simply a "constitutive" relation, which can only be fully justified upon investigation of the quantum mechanical analysis.

We now turn to synthesising the information that we have laid out above in a selfconsistent, relativistic, Lagrangian framework. Firstly, it is necessary to generalise the non-relativistic concept of the spin angular momentum $s$ to the relativistic domain. This procedure is carried out in many textbooks on electrodynamics (see, e.g., [12], pp. 556$560)$; we shall not add anything new. One simply defines a four-vector $S^{\alpha}$ such that, in the rest frame of the particle, it has vanishing zero-component $S^{0}$, and its three-vector part $\boldsymbol{S}$ is equal to the non-relativistic spin $\boldsymbol{s}$. Since, by definition, the three-vector part of the four-velocity, $\boldsymbol{U}$, vanishes in this frame, the identity

$$
S^{\alpha} U_{\alpha}=0
$$

shows that only three of the $S^{\alpha}$ are independent, as one would expect from the nonrelativistic case.

In a completely analogous way, one can generalise the non-relativistic angular velocity vector $\boldsymbol{\omega}$ and the moment of inertia tensor $\boldsymbol{I}$ to their relativistic counterparts $\omega^{\alpha}$ and $I^{\alpha \beta}$. The non-relativistic rotational "kinetic" Lagrangian (3) can then be written relativistically as

$$
L_{\mathrm{rot}}=\frac{1}{2} \omega^{\alpha} I_{\alpha \beta} \omega^{\beta}
$$

One might wonder, at this point, how a spin-half particle can have a "kinetic" term of rotation, when it is well known that there is no classical "rotating model" that represents 
spin angular momentum. The answer is as follows: the functional form of the spin kinetic Lagrangian must be retained in the classical limit, regardless of whether or not it corresponds to any particular "model" that one might dream up. In fact, it will be found that the quantities $\omega^{\alpha}$ and $I_{\alpha \beta}$ will completely disappear from the final equations of motion; the only remaining quantity will be the physically observable quantity $S^{\alpha}$. Thus, by what might arguably be considered a sleight of hand, we can analyse the system in question within Lagrangian mechanics, without recourse to the particular Newtonian models of refs [4,6] 8], nor the original heuristic (albeit brilliant) arguments of Thomas [2] and Bargmann, Michel and Telegdi [3].

Before we can write down the final expression for the Lagrangian we shall use, we must first "massage" the magnetic interaction Lagrangian (四) into a more suitable form. Our major task is to the interpret the spin tensor, $\sigma^{\alpha \beta}$, that the Dirac equation introduces. This is not a trivial task: translating between the spin vector that we have already defined, $S^{\alpha}$, and a spin tensor, requires the use of both the alternating tensor $\varepsilon^{\alpha \beta \mu \nu}$, and another fourvector. Often, in quantum mechanics, one uses the canonical momentum vector, $p^{\alpha}$, for this purpose; the resultant "spin" vector is known as the Pauli-Lubanski vector,

$$
W^{\alpha} \equiv \varepsilon^{\alpha \beta \mu \nu} p_{\beta} S_{\mu \nu}
$$

This quantity is, indeed, very useful in many situations. However, we are here considering Lagrangian mechanics; therefore, we should expect that mechanical momenta (or, in other words, generalised velocities) should be employed; canonical momenta belong to Hamiltonian dynamics. We therefore follow Jackson ( [12], p. 556) in using the four-velocity to define the transformation between the spin tensor and the spin vector, namely

$$
S^{\alpha}=\frac{1}{2} \varepsilon^{\alpha \beta \mu \nu} U_{\beta} S_{\mu \nu}
$$

It is straightforward to verify that the reverse transformation of (7) is given by

$$
S^{\alpha \beta}=\varepsilon^{\alpha \beta \mu \nu} U_{\mu} S_{\nu}
$$


Insering this into (四) and simplifying, we finally obtain our desired magnetic interaction Lagrangian,

$$
L_{\text {int }}=\mu^{\alpha} \widetilde{F}_{\alpha \beta} U^{\beta}
$$

where $\widetilde{F}_{\alpha \beta} \equiv \frac{1}{2} \varepsilon_{\alpha \beta}{ }^{\mu \nu} F_{\mu \nu}$ is the dual electromagnetic field strength tensor, and the magnetic moment four-vector, $\mu^{\alpha}$ is, as noted previously, considered to be a four-vector "embedded" in the instrinsic rotational coördinates of the particle, and hence is functionally dependent on the Euler angles, but not their derivatives.

Our complete Lagrangian is then assembled simply from the kinetic rotational term (5), the magnetic interaction term (9), and the standard translational kinetic and electric charge interaction terms:

$$
L=\frac{1}{2} m U^{\alpha} U_{\alpha}+\frac{1}{2} \omega^{\alpha} I_{\alpha \beta} \omega^{\beta}+q U^{\alpha} A_{\alpha}+\mu^{\alpha} \widetilde{F}_{\alpha \beta} U^{\beta} .
$$

It is this Lagrangian, and most particularly its functional form, that forms the basis of the following section.

\section{DERIVATION}

We now turn to the question of deriving, from the Euler-Lagrange equations, the equations of motion for the particle under study. (In the following, our language shall describe classical quantities in the same way that Newton would have done, but in reality we are referring to the expectation values of the corresponding quantum mechanical operators for the single particle in question.) The generalised coördinates for the particle, following the discussion of the previous section, are taken to be the four translational degrees of freedom $z^{\alpha}$, together with the three Euler angles describing the intrinsic "orientation" of the particle.

The mathematical manipulations necessary to obtain the seven Euler-Lagrange equations are in principle no different to those in everyday classical mechanical problems [11]. There is one subtlety, however, that enters into one's consideration of the spin vector $S^{\alpha}$ 
and the magnetic moment vector $\mu^{\alpha}$. By their very definition, these vectors are "tied" to the particle's four-velocity, in the sense that identities such as (团) always holds true. One must therefore be careful when defining their proper-time derivative: since they are, in effect, defined with respect to an accelerated frame of reference, there is a difference between taking the time-derivatives of the components of the vector, and the components of the time-derivatives of the vector, as General Relativity teaches us. In fact, the philosophical framework of General Relativity tells us that it is the latter that is the generally invariant, "covariant" derivative which should be used in the relativistic Euler-Lagrange equations; the former is simply the "partial" derivative. However, it is straightforward to verify that they can be related via

$$
\frac{d}{d \tau}\left(C^{\alpha}\right) \equiv \dot{C}^{\alpha}+U^{\alpha}\left(\dot{U}_{\beta} C^{\beta}\right)
$$

where $C^{\alpha}$ is any space-like vector that is orthogonal to the particle's four-velocity (such as $S^{\alpha}$ and $\mu^{\alpha}$ ), the left hand side denotes the "covariant" derivative, and $\dot{C}^{\alpha}$ the "partial" derivative, of such a vector. (The Thomas precession [13,2] is, in fact, just another way of expressing this "General Relativity" effect-namely, the non-vanishing commutator of Lorentz boosts.)

It is now relatively straightforward to apply the Euler-Lagrange equations to the Lagrangian (10). The three Euler angle degrees of freedom lead, just as in the non-relativistic case [11], to the torque equation of motion for the particle. With some algebra and simplification, they (together with the identity (4) and the relation (11)) lead to the four-vector equation of motion

$$
\dot{S}_{\alpha}+U_{\alpha} \dot{U}^{\beta} S_{\beta}=F_{\alpha \beta} \mu^{\beta}+U_{\alpha} \mu^{\nu} F_{\nu \beta} U^{\beta}
$$

It should come as no surprise that this equation is precisely that obtained by Bargmann, Michel and Telegdi (equation (6) of ref. [3]), before they simply substituted in the Lorentz force law for $\dot{U}_{\beta}$ (i.e. equation (7) of [3]). As was noted in section [, this equation-(12) - is true in general for any point-like object generating an external magnetic dipole field [4]. 
We now turn our attention to the four translational degrees of freedom of the particle. The Euler-Lagrange equations for these coördinates, for the same Lagrangian (10) as used above to generate the Bargmann-Michel-Telegdi equation, immediately yield the four-equation of motion

$$
\frac{d}{d \tau}\left(m U_{\alpha}\right)=q F_{\alpha \beta} U^{\beta}+U^{\beta} \mu^{\nu} \partial_{\nu} \widetilde{F}_{\alpha \beta}+\widetilde{F}_{\alpha \beta}\left(\dot{\mu}^{\beta}+U^{\beta} \dot{U}_{\nu} \mu^{\nu}\right)+\varepsilon_{\alpha \beta \mu \nu} \mu^{\beta} U^{\nu} J_{\mathrm{ext}}^{\mu} .
$$

The first term on the right side is, of course, simply the Lorentz force. The second and third terms, on the other hand, are infinitely more interesting. The second term represents the gradient forces on the magnetic dipole moment, i.e., in the non-relativistic limit, $(\boldsymbol{\mu} \cdot \boldsymbol{\nabla}) \boldsymbol{B}$. The third term represents a force of the type $-\dot{\boldsymbol{\mu}} \times \boldsymbol{E}$ in the non-relativistic limit. The reason that they are so interesting is that many authors have argued that precisely these terms should constitute the non-relativistic limit of the magnetic dipole force (see, e.g., [5] and references therein; also [4:4:8]). Here, we have obtained these terms from a relativistic Lagrangian directly, without need for assumptions other than those outlined in the previous section.

The last term in (13) is, as a matter of principle, much deeper, but in practice completely ignorable. It constitutes a contact force between the particle and the electromagnetic current that is generating the "external" field. In practice, such an interaction is usually ignored; however, upon further investigation, it is recognised that it is precisely this contact force that allows the expression (13) to otherwise resemble so closely the force on a "monopoleconstructed" dipole model [4.6, while on the other hand possessing an interaction energy equivalent to a "current loop" model [4,6 8] as required for agreement with atomic hyperfine levels [14]. It is in this way that the "Lagrangian-based" model of this paper seems to select those desirable properties of both the "monopole-constructed" and "current-loop" models, while being classically equivalent to neither.

Now that we have dealt with the dynamics of the Euler-Lagrange formalism, and have obtained allegedly appropriate equations of motion, we can now specify the exact "constitutive relation" between the magnetic moment and the spin of the particle, for the particular 
case we wish to study: a spin-half particle. From quantum mechanics, we know that the these two quantities are always parallel, namely,

$$
\mu^{\alpha}=\zeta S^{\alpha}
$$

where $\zeta \equiv \mu / s$ is some constant, a property of the particle in question, which, for particle of charge $q$, is commonly written in terms of the $g$ factor as $\zeta \equiv g q / 2 m$. Before we can use (14), however, we first note that equation (13) is itself in a somewhat awkward form: the right hand side involves both $\dot{\mu}^{\alpha}$ and $\dot{U}^{\alpha}$. However, the parallelism condition (14) allows us to uncouple equations (12) and (13) simply by substituting (12) into (13), yielding

$$
\frac{d}{d \tau}\left(m U_{\alpha}\right)=q F_{\alpha \beta} U^{\beta}+U^{\beta} \mu^{\nu} \partial_{\nu} \widetilde{F}_{\alpha \beta}+\zeta\left\{\widetilde{F}_{\alpha \beta} F^{\beta}{ }_{\nu} \mu^{\nu}+\widetilde{F}_{\alpha \beta} U^{\beta}\left(\mu^{\sigma} F_{\sigma \tau} U^{\tau}\right)\right\}
$$

where we have, for practical simplicity, dropped the contact term in (13).

There are now several important things we can do with equations (12) and (15). Most importantly, we examine how the mass of the particle changes with time; if it is not constant, then our equations of motion cannot possibly apply to any particle, such as an electron or muon, that has a constant rest mass. (It should be noted that the classical relativistic formalism we have employed has made no assumptions as to the constancy of the rest mass $m$ with time: in general, the mass may change as the system in question gains energy from or loses energy to the external field.) It is straightforward to verify that the general proper-time rate of change of the mass of a particle, $\dot{m}$, can be expressed as

$$
\dot{m} \equiv U^{\alpha} \frac{d}{d \tau}\left(m U_{\alpha}\right)
$$

Using the the identity (ब1), and the fact that $\widetilde{F}_{\alpha \beta} F^{\beta}{ }_{\nu}$ is proportional to the metric, $g_{\alpha \nu}$, it follows that (15) yields, in (16), $\dot{m}=0$. In other words, the set of equations (12) and (15) rigorously maintain the constancy of the mass of the particle. This property is far from trivial; for example, in a classic textbook ( [15], p. 74), a time-varying "effective mass" of the electron was introduced, due to the fact that the equations of motion derived therein allowed time-changing rest masses. It is of utmost importance that this difficulty-present 
in most previous attempts at consistent equations of motion for a dipole - is overcome in the Lagrangian treatment of this paper.

We now verify that the the parallelism condition, (14), is consistent with the equations of motion (12) and (15). The reason for this concern is that we have assumed that the magnetic moment is a constant-magnitude vector, whereas the spin itself is a dynamical quantity that may, in general, change its magnitude. We must verify that, if (14) is assumed to hold true at one particular proper time $\tau$, the equation of motion (12) does not change the magnitude of the spin. On diffentiation of the identity $s^{2} \equiv-S^{\alpha} S_{\alpha}$, one finds $\dot{s}=-\frac{1}{2} \dot{S}^{\alpha} S_{\alpha}$; use of (14) in (12) then shows that (12) does, in fact, yield $\dot{s}=0$. Thus, the magnitude of the spin is a rigorous constant of the motion, just as is the mass.

Our work is now essentially complete. However, to make practical use of the equations (12) and (15), it is appropriate to both present them in a more computationally-friendly form, and to highlight clearly where they add to existing knowledge of spin-half particles. These two tasks can essentially be carried out simultaneously. We shall transform (12) and (15) into the standard " $3+1$ " form, as, for example, presented in Jackson's textbook ( [12], pp. 556-560). This involves using the three-velocity of the particle in some particular fixed "lab" frame, $\boldsymbol{v}$ (Jackson uses the symbol $\boldsymbol{\beta}$ ), as well as the three-spin $\boldsymbol{s}$ of the particle as seen in its rest frame, but referred to the (non-rotating) coördinates of the "lab" frame. The procedure used to effect this transformation is described in detail in [12]; the algebra is tedious, but straightforward. The results for equations (15) and (12) are

$$
\frac{d \boldsymbol{v}}{d t}=\frac{q}{\gamma m} \boldsymbol{E}^{\prime \prime}+\frac{g_{\mathrm{eff}}}{\gamma m} \boldsymbol{B}^{\prime \prime}+\frac{\Theta}{\gamma^{2} m} \boldsymbol{s}^{\prime}
$$

and

$$
\frac{d s}{d t}=s \times \Omega_{\text {new }},
$$

where

$$
\begin{aligned}
\boldsymbol{\Omega}_{\mathrm{new}}= & \left\{\zeta-\frac{\gamma-1}{\gamma} \frac{q}{m}\right\} \boldsymbol{B}-\left\{\zeta-\frac{\gamma}{\gamma+1} \frac{q}{m}\right\} \boldsymbol{v} \times \boldsymbol{E}-\frac{\gamma}{\gamma+1}\left\{\zeta-\frac{q}{m}\right\}(\boldsymbol{v} \cdot \boldsymbol{B}) \boldsymbol{v} \\
& +\frac{\gamma-1}{\gamma} \frac{g_{\mathrm{eff}}}{m} \boldsymbol{E}+\frac{\gamma}{\gamma+1} \frac{g_{\mathrm{eff}}}{m} \boldsymbol{v} \times \boldsymbol{B}-\frac{\gamma}{\gamma+1} \frac{g_{\mathrm{eff}}}{m}(\boldsymbol{v} \cdot \boldsymbol{E}) \boldsymbol{v}+\frac{\Theta}{m(\gamma+1)} \boldsymbol{v} \times \boldsymbol{s}
\end{aligned}
$$


and we have defined the convenient quantities

$$
\begin{aligned}
\zeta & \equiv \frac{\mu}{s} \\
\Theta & \equiv \zeta^{2}(\boldsymbol{E} \cdot \boldsymbol{B}), \\
\partial^{\prime} & \equiv \frac{\partial}{\partial t}+\frac{\gamma}{\gamma+1}(\boldsymbol{v} \cdot \boldsymbol{\nabla}), \\
\boldsymbol{s}^{\prime} & \equiv \boldsymbol{s}-\frac{\gamma}{\gamma+1}(\boldsymbol{v} \cdot \boldsymbol{s}) \boldsymbol{v} \\
\boldsymbol{E}^{\prime} & \equiv \boldsymbol{E}+\boldsymbol{v} \times \boldsymbol{B}-\frac{\gamma}{\gamma+1}(\boldsymbol{v} \cdot \boldsymbol{E}) \boldsymbol{v}, \\
\boldsymbol{E}^{\prime \prime} & \equiv \boldsymbol{E}+\boldsymbol{v} \times \boldsymbol{B}-(\boldsymbol{v} \cdot \boldsymbol{E}) \boldsymbol{v}, \\
\boldsymbol{B}^{\prime \prime} & \equiv \boldsymbol{B}-\boldsymbol{v} \times \boldsymbol{E}-(\boldsymbol{v} \cdot \boldsymbol{B}) \boldsymbol{v}, \\
g_{\mathrm{eff}} & \equiv \zeta(\boldsymbol{s} \cdot \boldsymbol{\nabla})+\gamma \zeta(\boldsymbol{s} \cdot \boldsymbol{v}) \partial^{\prime}-\gamma \zeta^{2}\left(\boldsymbol{s} \cdot \boldsymbol{E}^{\prime}\right),
\end{aligned}
$$

and, in all expressions, the partial derivatives act only on the external field quantities $\boldsymbol{E}$ and $\boldsymbol{B}$.

For ease of comparison with the equations of motion in current usage, we present the Lorentz force law in the same form as (17),

$$
\frac{d \boldsymbol{v}}{d t}=\frac{q}{\gamma m} \boldsymbol{E}^{\prime \prime}
$$

and, likewise, the precession frequency vector for the Thomas spin equation ( [12], p. 559):

$$
\boldsymbol{\Omega}_{\mathrm{old}}=\left\{\zeta-\frac{\gamma-1}{\gamma} \frac{q}{m}\right\} \boldsymbol{B}-\left\{\zeta-\frac{\gamma}{\gamma+1} \frac{q}{m}\right\} \boldsymbol{v} \times \boldsymbol{E}-\frac{\gamma}{\gamma+1}\left\{\zeta-\frac{q}{m}\right\}(\boldsymbol{v} \cdot \boldsymbol{B}) \boldsymbol{v} .
$$

It can be seen that, as advertised, the Lorentz and Thomas equations are contained completely in the new equations. However, several new features are present in both the new force equation, (17), and the new precession frequency vector, $\boldsymbol{\Omega}_{\text {new }}$. Most obviously, the magnetic dipole force is now included in (17), albeit somewhat obscured by the multitude of "convenient quantities" introduced for typographical sanity. A recognition of this expression may again be obtained by taking the non-relativistic limit (first order in $\boldsymbol{v}$, ignoring Thomas precession); (17) then returns us to

$$
\frac{d}{d t}(m \boldsymbol{v})=q(\boldsymbol{E}+\boldsymbol{v} \times \boldsymbol{B})+(\boldsymbol{\mu} \cdot \boldsymbol{\nabla})(\boldsymbol{B}-\boldsymbol{v} \times \boldsymbol{E})-\dot{\boldsymbol{\mu}} \times \boldsymbol{E},
$$


which is, as noted earlier, the now generally-accepted [5.74] dipole force expression. There are, of course, numerous new subtleties of (17) that arise from relativistic kinematics; we shall, however, defer a more exhaustive investigation of them to another place.

Turning, now, to the spin precession equation (18), what may come as a surprise to some is that there are differences between $\boldsymbol{\Omega}_{\mathrm{old}}$ and $\boldsymbol{\Omega}_{\text {new }}$. The reason, however, is simply found: the Thomas precession effects in the spin precession equation depend on the acceleration of the particle; clearly, if we have obtained a more accurate force law, then these changes will necessarily feed through to the spin equation as well. It is interesting to note that the approximate nature of the Thomas expression $\boldsymbol{\Omega}_{\text {old }}$ was noted explicitly by Thomas himself [2], and by Bargmann, Michel and Telegdi in their re-derivation [3], but that, in the intervening decades, this approximate nature has been lost on many practitioners, who mistakenly believe $\boldsymbol{\Omega}_{\text {old }}$ to be an exact expression.

At this point, it is worthwhile commenting on the absence of any presence of radiation reaction forces in the equations that have been derived above. Their omission can, in fact, be traced back directly to the (implicit) assumption that the electromagnetic potentials and fields in the Lagrangian (101) are the externally generated ones only. In the case of the electric charge interaction, it was recognised by Lorentz [16], and later clearly explained by Heitler [17], that this is an incorrect assumption. For full consistency, one must include the potentials and fields of the particle itself in the Lagrangian, even though they appear, at first sight, to be hopelessly divergent quantities. If one proceeds with extreme care, one can show that the effects on the equations of motion of this "self-interaction" are, in fact, twofold: firstly, the addition of the (finite) radiation reaction terms of the Lorentz-Dirac equation; and, secondly, the dynamical effects explaining the electromagnetic self-energy contributions to the rest mass of the system, for whatever arbitrary charge distribution is assumed. The beauty of this procedure is that it reveals the all-encompassing nature of the Lagrangian; no further input is required to obtain the equations of motion.

The success of the above procedure clearly indicates that a similar procedure should be undertaken for the additional magnetic moment interaction Lagrangian present in (10). 
Barut and Unal [10] have considered the dipole radiation reaction question from the point of view of a semi-classical Zitterbewegung model, but to our knowledge an exact treatment of this problem in terms of the classical spin vector $s$ has not been performed. This problem is one that we are currently investigating. However, it is vastly more complicated than the electric charge case, by virtue of the inclusion of rotatonal degrees of freedom for the particle. In addition, one already knows in advance that the electric charge and magnetic moment interact in their respective radiative terms, as is evidenced by the Sokolov-Ternov effect [18 (the polarisation of electrons due to their emitted synchrotron radiation), spectacularly confirmed in the polarisation experiments at LEP in recent years [19,20. Any prospective solution of the combined radiation reaction equations for a charged, spinning particle must therefore, at the very least, reproduce this important result.

\section{CONCLUSIONS}

It has been shown, in this paper, that it is possible to construct a fully consistent, comprehensive, relativistic classical Lagrangian framework for analysing the motion of spinning particles possessing both electric charge and magnetic dipole moments. The results obtained here are not revolutionary. They encompass the well-known Lorentz force and Thomas-Bargmann-Michel-Telegdi equations. They provide a rigorous foundation for the magnetic dipole force law currently believed to be the most appropriate for such particles. They further integrate, seamlessly, this dipole force with the Lorentz force and ThomasBargmann-Michel-Telegdi equations, in a fully relativistic way.

It should be noted that the results of this paper agree with the lowest-order terms obtained in the analysis of Anandan, based on the Dirac equation [21] - and, of particular note, the "Anandan force" proportional to $\boldsymbol{E} \times(\boldsymbol{\mu} \times \boldsymbol{B})$, which is, of course, simply the zeroth-order term in the force term $-\dot{\boldsymbol{\mu}} \times \boldsymbol{E}$ above, when it realised that $\dot{\boldsymbol{\mu}}$ is, via the ThomasBMT equation, proportional to $\boldsymbol{\mu} \times \boldsymbol{B}$ to zeroth order. The current work, however, includes

terms to all orders in the particle's velocity, not just the lowest-order limit of Anandan. It 
should also be noted that the criticisms of Casella and Werner [22] of Anandan's analysis 21] are erroneous, being based on an obvious omission of all "spin-flip" terms from their quantum mechanical equations of motion.

The framework outlined in this paper may now be used as a platform for full inclusion of radiation reaction - not just for the electric charge, but also for the magnetic moment, and their mutual interactions - in the classical limit. If the enormous assistance provided by the existing classical radiation reaction theory, both in terrestrial and astrophysical applications, is any guide, then one can only speculate what additional richness of physical phenomena will be made sensible with this addition to our analytical resources.

\section{ACKNOWLEDGMENTS}

Helpful discussions with I. Khriplovich, J. Anandan, N. Mukhopadhyay, L. Wilets, P. Herczeg, A. G. Klein, G. I. Opat, J. W. G. Wignall, A. J. Davies, G. N. Taylor, K. Jones, R. E. Behrend and S. Bass are gratefully acknowledged. This work was supported in part by the Australian Research Council, an Australian Postgraduate Research Allowance and a Dixson Research Scholarship. We warmly thank the Institute for Nuclear Theory at the University of Washington for its hospitality and the United States Department of Energy Grant \#DOE/ER40561 for partial support during the completion of this work. 


\section{REFERENCES}

[1] P. A. M. Dirac, The Principles of Quantum Mechanics, 4th ed. (Oxford University Press, Oxford, 1958).

[2] L. H. Thomas, Phil. Mag. 3 (1927) 1.

[3] V. Bargmann, L. Michel and V. L. Telegdi, Phys. Rev. Lett. 2 (1959) 435.

[4] P. Hraskó, Nuovo Cimento B 3 (1971) 213.

[5] S. R. Barone, Phys. Rev. D 8 (1973) 3492.

[6] T. H. Boyer, Phys. Rev. A 36 (1987) 5083.

[7] Y. Aharonov, P. Pearle and L. Vaidman, Phys. Rev. A 37 (1988) 4052.

[8] A. S. Goldhaber, Phys. Rev. Lett. 62 (1989) 482.

[9] Y. Aharonov and A. Casher, Phys. Rev. Lett. 53 (1984) 319.

[10] A. O. Barut and N. Unal, Phys. Rev. A 40 (1989) 5404.

[11] H. Goldstein, Classical Mechanics, 2nd ed. (Addison-Wesley, Massachusetts, 1980).

[12] J. D. Jackson, Classical Electrodynamics, 2nd ed. (John Wiley \& Sons, New York, 1975).

[13] L. H. Thomas, Nature (10 April 1926) 514.

[14] G. I. Opat, Phys. Lett. B 60 (1976) 205.

[15] A. O. Barut, Electrodynamics and Classical Theory of Particles and Fields (Macmillan, 1964).

[16] H. A. Lorentz, The Theory of Electrons (Dover, New York, 1952).

[17] W. Heitler, The Quantum Theory of Radiation, 3rd ed. (Oxford University Press, London, 1954).

[18] A. A. Sokolov and I. M. Ternov, Dokl. Akad. Nauk SSSR 153 (1963) 1052. 
[19] L. Knudsen et al., Phys. Lett. B 270 (1991) 97.

[20] L. Arnaudon et al., Phys. Lett. B 284 (1992) 431.

[21] J. Anandan, Phys. Lett. A 138 (1989) 347; Proc. 3rd Int. Symp. Foundations of Quantum Mechanics (Tokyo, 1989) 98.

[22] R. C. Casella and S. A. Werner, Phys. Rev. Lett. 69 (1992) 1625. 